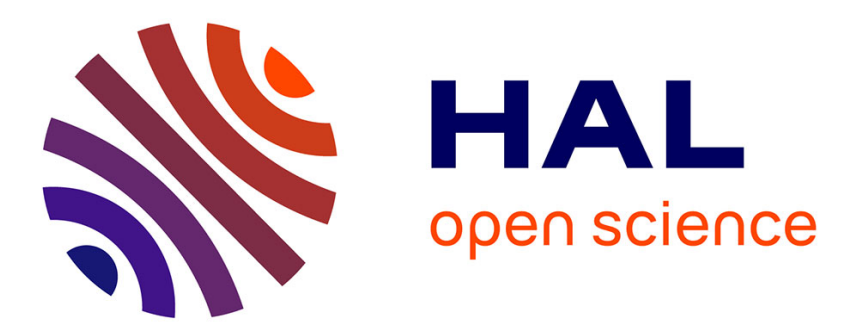

\title{
Dynamic Boundary Stabilization of Linear Parameter Varying Hyperbolic Systems: Application to a Poiseuille Flow
}

Felipe Castillo Buenaventura, Emmanuel Witrant, Luc Dugard

\section{- To cite this version:}

Felipe Castillo Buenaventura, Emmanuel Witrant, Luc Dugard. Dynamic Boundary Stabilization of Linear Parameter Varying Hyperbolic Systems: Application to a Poiseuille Flow. IFAC Joint conference SSSC - 11th Workshop on Time-Delay Systems, Feb 2013, Grenoble, France. hal-00752313

\section{HAL Id: hal-00752313 \\ https://hal.science/hal-00752313}

Submitted on 15 Nov 2012

HAL is a multi-disciplinary open access archive for the deposit and dissemination of scientific research documents, whether they are published or not. The documents may come from teaching and research institutions in France or abroad, or from public or private research centers.
L'archive ouverte pluridisciplinaire HAL, est destinée au dépôt et à la diffusion de documents scientifiques de niveau recherche, publiés ou non, émanant des établissements d'enseignement et de recherche français ou étrangers, des laboratoires publics ou privés. 


\title{
Dynamic Boundary Stabilization of Linear Parameter Varying Hyperbolic Systems: Application to a Poiseuille Flow
}

\author{
F. Castillo*, E. Witrant and ${ }^{*}$ L. Dugard ${ }^{*}$ \\ * UJF-Grenoble1/CNRS, Grenoble Image Parole Signal Automatique \\ (GIPSA-Lab), UMR5216, B.P. 46, 38402 St Martin d'Heres, France \\ (e-mail: felipe.castillo-buenaventura, \\ emmanuel.witrant,luc.dugard@gipsa-lab.fr)
}

\begin{abstract}
The problem of boundary control in first order linear parameter varying (LPV) hyperbolic systems with dynamics associated with the boundary conditions is considered in this article. By means of Lyapunov based techniques, some sufficient conditions are derived for the exponential stability of these infinite dimensional systems. A polytopic approach is developed in order to synthesize a robust boundary control which guarantees the exponential stability for a given convex parameter set. An application using a Poiseuille flow control experimental setup illustrates the main results.
\end{abstract}

Keywords: Hyperbolic Systems; boundary control, LMI.

\section{INTRODUCTION}

Fluid transport is a phenomenon often encountered in many industrial applications of significant importance such as hydraulic networks (Santos and Prieur [2008]), gas flow in pipelines (Bastin et al. [2008]) and flow regulation in deep pits (Witrant et al. [Jan. 2010]), among others. These kind of infinite dimensional systems introduce variable time-delays that make the closed loop control much more challenging. The fluid transport is often modeled by balance laws which are hyperbolic partial differential equations (PDE), commonly used to express the fundamental dynamics of open conservative systems. Measurements and actuators in distributed parameter systems are not usually available. It is more common for them to be located at the boundaries which is particularly true in applications such as the ones previously mentioned.

The stability problem of the boundary control in time invariant hyperbolic systems has been considered for a long time in the literature, as reported in Coron et al. [2007], Coron et al. [2008] and Prieur et al. [2008], among other references. However, the boundary control of LPV hyperbolic systems has not been found in the literature, which is the principal concern of this work. Also, most results in boundary control of hyperbolic systems consider that the control can act directly at the boundary conditions. More precisely, no time response limitation is considered at the boundary actuator. This is a valid assumption in applications such as the ones addressed in Bastin et al. [2009], where the wave travel time can be considered much longer than the actuator time response, allowing a static relationship between the control input and the boundary condition to be established. Nevertheless, there are applications where the dynamics associated with the boundary control cannot be neglected. To address this problem, a discretization of the infinite-dimensional system has been used in order to apply finite-dimensional control tools such as in Castillo et al. [2012]. In Castillo et al. [2013], some sufficient conditions for the exponential stability of hyperbolic systems with LTI dynamic boundary conditions have been given.

The present work focuses on the stability problem of linear parameter varying hyperbolic systems in presence of known LPV dynamics at the boundary conditions. To demonstrate asymptotic stability of this kind of hyperbolic systems, Riemann coordinates are used along with an extension of the strict Lyapunov function formulation presented in Coron et al. [2007]. Sufficient conditions are derived on the system in terms of the boundary conditions to prove Lyapunov stability. The sufficient conditions for stability are presented in a linear matrix inequality (LMI) framework. An example of a physical experimental setup specifically designed to study the fluid transport phenomenon is developed in detail to illustrate the proposed boundary control strategy.

This paper is organized as follows: in Section 2, the class of hyperbolic systems under consideration is given and the control problem is presented. In Section 3, the main stability results for linear parameter varying hyperbolic systems with dynamic behavior at the boundary conditions are presented. In Section 4, a reduced physical model of an experimental setup for the regulation of the output temperature in a Poiseuille flow is developed. Finally, in Section 5, a temperature boundary control is designed for the experimental setup using the main contributions of this work. Simulations results are presented to illustrate the effectiveness of the proposed control strategy. 


\section{PROBLEM FORMULATION}

This section gives a brief introduction on first order parameter-varying linear hyperbolic systems in one dimension space and presents the specific hyperbolic systems considered in this work. Let $n$ be a positive integer, $\Theta$ an open non-empty convex set of $\mathbb{R}^{n}$ and $Z_{\varphi}$ a non empty convex set of $\mathbb{R}^{l}$. Consider the general class of first order linear parameter-varying hyperbolic systems in Riemann coordinates of order $n$ defined as follows:

$$
\partial_{t} \xi(x, t)+\Lambda(\varphi) \partial_{x} \xi(x, t)=0 \quad \forall x \in[0,1], t \geq 0
$$

where $\xi:[0,1] \times \mathbb{R}^{n} \rightarrow \Theta, \varphi$ is a varying parameter vector that takes values in the parameter space $Z_{\varphi}$, $\Lambda(\varphi): Z_{\varphi} \rightarrow \mathbb{R}^{n \times n}$ is a diagonal and invertible matrix function (called the characteristic matrix) such that $\Lambda(\varphi)=\operatorname{diag}\left(\lambda_{1}(\varphi), \lambda_{2}(\varphi), \ldots, \lambda_{n}(\varphi)\right)$ with:

$$
\begin{aligned}
& \lambda_{1}(\varphi)<\ldots<\lambda_{m}(\varphi)<0<\lambda_{m+1}(\varphi)<\ldots<\lambda_{n}(\varphi) \\
& \forall \varphi \in Z_{\varphi}
\end{aligned}
$$

The state description can be partitioned as: $\xi=\left[\begin{array}{l}\xi_{-} \\ \xi_{+}\end{array}\right]$ where $\xi_{-}$is in $\mathbb{R}^{m}$ and $\xi_{+}$is in $\mathbb{R}^{n-m}$. Let define:

$$
\Lambda^{+}(\varphi)=\operatorname{diag}\left(\left|\lambda_{1}(\varphi)\right|,\left|\lambda_{2}(\varphi)\right|, \ldots,\left|\lambda_{n}(\varphi)\right|\right)
$$

Considering the system (1), it is possible to introduce the change of variable $\left(\begin{array}{c}\xi_{-}(1-x, t) \\ \xi_{+}(x, t)\end{array}\right)$ to obtain a PDE of the form (1), whose corresponding diagonal characteristic matrix function is $\Lambda^{+}(\varphi)$. Therefore, it can be assumed without loss of generality that $m=0$ and $\Lambda(\varphi)=\Lambda^{+}(\varphi)$. In this work, the following dynamic boundary condition for (1) is considered.

$$
\begin{aligned}
& \dot{X}_{c}=A_{c}(\varphi) X_{c}+B_{c}(\varphi) u \\
& Y_{c}=X_{c}
\end{aligned}
$$

with

$$
X_{c}=\left(\begin{array}{c}
\xi_{-}(1, t) \\
\xi_{+}(0, t)
\end{array}\right), \quad u=K Y_{\xi}, \quad Y_{\xi}=\left(\begin{array}{c}
\xi_{-}(0, t) \\
\xi_{+}(1, t)
\end{array}\right)
$$

where $A_{c}: Z_{\varphi} \rightarrow \mathbb{R}^{n \times n}, B_{c}: Z_{\varphi} \rightarrow \mathbb{R}^{n \times n}, K \in \mathbb{R}^{n \times n}$ and $u \in \mathbb{R}^{n}$. Given a continuously differentiable function $\xi^{0}:[0,1] \rightarrow \Theta$ that satisfies the zero-order and oneorder compatibility conditions (required for existence of an unique $C^{1}$ solution), then the initial condition can be defined as (see Kmit [2008]):

$$
\xi(x, 0)=\xi^{0}(x), \quad \forall x \in[0,1]
$$

The control problem under consideration in this work is to find a control gain $K$ such that system (1) with boundary condition (4) - (5) is Lyapunov stable $\forall \varphi \in Z_{\varphi}$.

\section{STABILITY OF PARAMETER-VARYING LINEAR HYPERBOLIC SYSTEMS WITH DYNAMIC BOUNDARY CONDITIONS}

Consider the following polytopic linear representation of the parameter varying characteristic matrix:

$$
\Lambda(\varphi)=\sum_{i=1}^{2^{l}} \alpha_{i}(\varphi) \Lambda\left(w_{i}\right)
$$

$\forall \varphi \in Z_{\varphi}$, where $\varphi$ is a varying parameter vector that takes values in the parameter space $Z_{\varphi}$ defined by (see Angelis [2001]):

$$
Z_{\varphi}:=\left\{\left[\varphi_{1}, \ldots, \varphi_{l}\right]^{T} \in \mathbb{R}^{l}, \varphi_{i} \in\left[\bar{\varphi}_{i}, \underline{\varphi}_{i}\right], \forall i=1 \ldots l\right\}
$$

where $l$ is the number of varying parameters, $\alpha_{i}(\varphi)$ is a scheduling function $\alpha_{i}: Z_{\varphi} \rightarrow[0,1], w_{i} \in Z_{\varphi}$ are the $2^{l}=N_{\varphi}$ vertices of the polytope formed by all extremities $\left(\bar{\varphi}_{i}\right.$ and $\left.\underline{\varphi}_{i}\right)$ of each varying parameter $\varphi \in Z_{\varphi}$ and $\sum_{i=1}^{2^{l}} \alpha_{i}(\varphi) \Lambda\left(w_{i}\right): Z_{\varphi} \rightarrow \mathbb{R}^{n \times n}$. In general, all the admissible values of the vector $\varphi$ are constrained in an hyperrectangle in the parameter space $Z_{\varphi}$. The scheduling functions $\alpha_{i}(\varphi)$ are defined as:

$$
\alpha_{i}(\varphi)=\frac{\prod_{k=1}^{l}\left|\varphi_{k}-C\left(w_{i}\right)_{k}\right|}{\prod_{k=1}^{l}\left|\bar{\varphi}_{k}-\underline{\varphi}_{k}\right|}
$$

where:

$$
\begin{aligned}
C\left(w_{i}\right)_{k}= & \left\{\varphi_{k} \mid \varphi_{k}=\bar{\varphi}_{k} \text { if }\left(w_{i}\right)_{k}=\underline{\varphi}_{k}\right. \\
& \text { or } \left.\varphi_{k}=\underline{\varphi}_{k} \text { otherwise }\right\}
\end{aligned}
$$

which exhibits the following properties:

$$
\alpha_{i}(\varphi) \geq 0, \quad \sum_{i=1}^{N_{\varphi}} \alpha_{i}(\varphi)=1
$$

Consider (1) as an equivalent parameter varying hyperbolic system defined by:

$$
\begin{aligned}
& \partial_{t} \xi(x, t)+\sum_{i=1}^{N_{\varphi}} \alpha_{i}(\varphi) \Lambda\left(w_{i}\right) \partial_{x} \xi(x, t)=0 \\
& \forall \varphi \in Z_{\varphi}, \quad \forall x \in[0,1], \quad t \geq 0
\end{aligned}
$$

with boundary conditions

$$
\begin{aligned}
& \dot{X}_{c}=\sum_{i=1}^{N_{\varphi}} \alpha_{i}(\varphi) A_{c}\left(w_{i}\right) X_{c}+\sum_{i=1}^{N_{\varphi}} \alpha_{i}(\varphi) B_{c}\left(w_{i}\right) u \\
& Y_{c}=X_{c}
\end{aligned}
$$

Using (12) along with the boundary conditions (13), the following theorem states some sufficient conditions to ensure exponential stability for system (1) with boundary 
conditions (4) and initial condition (6) $\forall \varphi \in Z_{\varphi}$.

Theorem 1. Consider the system (1) with boundary conditions (4) and initial conditions (6). Assume that there exists a diagonal positive definite matrix $Q \in \mathbb{R}^{n \times n}$ such that the following $L M I$ is satisfied $\forall i \in\left[1, \ldots, N_{\varphi}\right]$

$$
\left[\begin{array}{cc}
Q A_{c}\left(w_{i}\right)^{T}+A_{c}\left(w_{i}\right) Q+\Lambda^{+}\left(w_{i}\right) Q & B_{c}\left(w_{i}\right) Y \\
Y^{T} B_{c}\left(w_{i}\right)^{T} & -\Lambda^{+}\left(w_{i}\right) Q
\end{array}\right] \prec 0
$$

where $Y=K Q$, then there exist two constants $\alpha>0$ and $M>0$ such that, for all continuously differentiable functions $\xi^{0}:[0,1] \rightarrow \Theta$ satisfying the zero-order and oneorder compatibility conditions, the solution of (1), (4) and (6) satisfies for all $t \geq 0$

$$
\begin{aligned}
& \left\|X_{c}(t)\right\|^{2}+\|\xi(x, t)\|_{L^{2}(0,1)} \leq \\
& M e^{-\alpha t}\left(\left\|X_{c}(0)\right\|^{2}+\left\|\xi^{0}(x)\right\|_{L^{2}(0,1)}\right)
\end{aligned}
$$

Proof Given a diagonal positive definite matrix $P$, consider, as an extension of the Lyapunov function proposed in Coron et al. [2007], the quadratic strict Lyapunov function candidate defined for all continuously differentiable functions $\xi:[0,1] \rightarrow \Theta$ as:

$$
V\left(\xi, X_{c}\right)=X_{c}^{T} P X_{c}+\int_{0}^{1}\left(\xi^{T} P \xi\right) e^{-\mu x} d x
$$

where $\mu$ is a positive scalar that will be precised below. Computing the time derivative of $V$ along the classical $C^{1}$ solutions of (1) with boundary conditions (4) and initial conditions (6), yields the following:

$$
\dot{V}=\dot{X}_{c}^{T} P X_{c}+X_{c}^{T} P \dot{X}_{c}+\int_{0}^{1}\left(\dot{\xi}^{T} P \xi+\xi^{T} P \dot{\xi}\right) e^{-\mu x} d x
$$

After integration by parts the following is obtained:

$$
\begin{aligned}
& \dot{V}=\sum_{i=1}^{2^{l}} \alpha_{i}(\varphi)\left[\left(X_{c}^{T}\left(A_{c}\left(w_{i}\right)^{T} P+P A_{c}\left(w_{i}\right)\right) X_{c}\right)\right. \\
& +\left(Y_{\xi}^{T} K^{T} B_{c}\left(w_{i}\right)^{T} P X_{c}+X_{c}^{T} P B_{c}\left(w_{i}\right) K Y_{\xi}\right) \\
& -\left.\left[e^{-\mu x} \xi^{T} \Lambda\left(w_{i}\right) P \xi\right]\right|_{0} ^{1} \\
& \left.\left.-\mu \int_{0}^{1}\left(\xi^{T}\right) \Lambda\left(w_{i}\right) P \xi\right) e^{-\mu x} d x\right]
\end{aligned}
$$

The previous equation can be written in terms of the boundary conditions as follows:

$$
\begin{aligned}
& \dot{V}=\sum_{i=1}^{2^{l}} \alpha_{i}(\varphi)\left[\left[-\mu X_{c}^{T} \Lambda\left(w_{i}\right) P X_{c}\right.\right. \\
& -\mu \int_{0}^{1}\left(\xi^{T} \Lambda\left(w_{i}\right) P \xi\right) e^{-\mu x} d x \\
& \left.+\left[\begin{array}{c}
X_{c} \\
Y_{\xi}
\end{array}\right]^{T}\left[\begin{array}{cc}
A\left(w_{i}\right)^{T} P+P A\left(w_{i}\right) & P B_{c}\left(w_{i}\right) K \\
+\Lambda\left(w_{i}\right) P+\mu \Lambda\left(w_{i}\right) P & \\
K^{T} B_{c}\left(w_{i}\right)^{T} P & -e^{-\mu} \Lambda\left(w_{i}\right) P
\end{array}\right]\left[\begin{array}{c}
X_{c} \\
Y_{\xi}
\end{array}\right]\right]
\end{aligned}
$$

Note that (14) is equivalent to consider that:

$$
\left[\begin{array}{cc}
A\left(w_{i}\right)^{T} P+P A\left(w_{i}\right)+\Lambda P & P B_{c}\left(w_{i}\right) K \\
K^{T} B_{c}\left(w_{i}\right)^{T} P & -\Lambda\left(w_{i}\right) P
\end{array}\right] \prec 0
$$

which is obtained by multiplying both sides of (20) by $\operatorname{diag}\left(P^{-1}, P^{-1}\right)$ and performing the variable transformations $Q=P^{-1}$ and $Y=K Q$. Thus, for a small enough and positive $\mu$ and the fact that by definition, $\sum_{i=1}^{2^{l}} \alpha_{i}(\varphi)=1$ and $\alpha_{i} \geq 0$, the third term of (19) is always negative. It is clear that there exists an $\epsilon$ such that $\Lambda\left(w_{i}\right)>\epsilon I^{n \times n}$ (e.g $\epsilon$ could be the smallest eigenvalue of $\left.\Lambda\left(w_{i}\right) \forall i \in\left[1, \ldots, N_{\varphi}\right]\right)$. This implies that:

$$
\dot{V} \leq-\mu \epsilon V\left(\xi, X_{c}\right)
$$

Therefore for a sufficiently small $\mu>0$, the function (16) is a Lyapunov function for the hyperbolic system (1) with boundary conditions (4).

\section{EXPERIMENTAL SETUP MODELING}

To further investigate the phenomenon of fluid transport in a Poiseuille flow with dynamics at the boundary conditions, an experimental setup has been designed to test and validate advanced control strategies. Figure 1 shows the schematic of the proposed device.

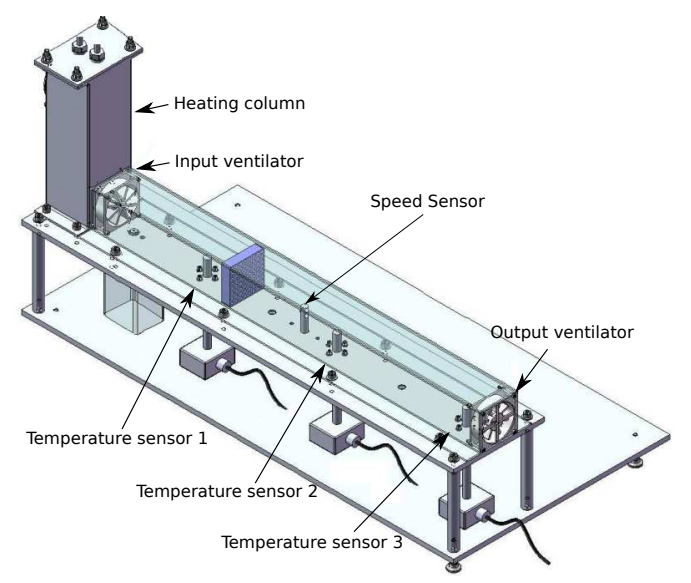

Fig. 1. Schematic of the experimental setup

This device mainly consists of a heating column encasing a resistor, a tube, two ventilators, a gas speed meter and distributed temperature sensors. The control problem is to regulate the outlet temperature of the tube by driving 
the power dissipated on the heating resistor at different air flow speeds (exogenous inputs produced by fans) through the tube. Only the outlet temperature and the flow speed will be considered as measurements for the closed loop boundary control strategy.

The modeling of the experimental setup presented in Figure 1 is done by considering two subsystems: the heating column and the tube. A zero-dimensional model (0-D) (control volume approach with heat exchanges coming from the heating resistor) represents the heating column. For the tube, a one dimensional (1-D) transport model is used to describe the gas density variations in the tube.

\subsection{Heating Column Model}

Figure 2 presents the schematic of the 0-D control volume approach considered for the heating column.

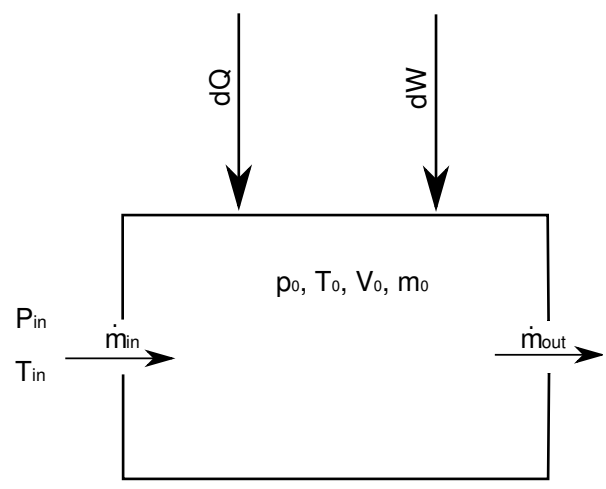

Fig. 2. Schematic of the control volume approach

Consider the internal energy of a perfect gas:

$$
U_{0}=C_{v} m_{0} T_{0}
$$

where $U_{0}$ is the gas internal energy, $T_{0}$ the gas temperature, $m_{0}$ the mass inside the column and $C_{v}$ the specific heat of the gas for constant volume. The time derivative of $(22)$ is:

$$
\dot{U}_{0}=C_{v} m_{0} \dot{T}_{0}+C_{v} T_{0} \dot{m}_{0}
$$

Using the first law of thermodynamics, the dynamics of the internal energy of the gas inside the column can also be given by:

$$
\dot{U}_{0}=\sum h_{i} \dot{m}_{i}+d Q+d W
$$

where $h_{i}$ is the specific enthalpy getting in and out of the volume with a mass flow rate $\dot{m}_{i}, d Q$ quantifies the heat exchanges and $d W$ is the work done by the gas. In the case of the heating column, there are two flows interacting with the volume, the input mass flow rate $\dot{m}_{i n}$ and the output mass flow rate $\dot{m}_{\text {out }}$. As the gas does not perform any work, then $d W=0$. In order to write (24) in terms of temperature, the specific enthalpy of a gas, defined by $h=C_{p} T$, where $C_{p}$ is the specific heat constant at constant pressure, is used. Therefore, $(24)$ can be expressed as:

$$
\dot{U}_{0}=C_{p} T_{i n} \dot{m}_{i n}-C_{p} T_{0} \dot{m}_{\text {out }}+d Q
$$

where $T_{\text {in }}$ is the heating column input temperature. To simplify the model, consider the following two hypotheses:

H-1 The pressure dynamics is much faster than the temperature dynamics, which allows a quasi-static behavior of the mass and pressure to be considered;

H-2 $p_{0} \approx p_{i n}$, where $p_{i n}$ is the input pressure;

Hypothesis H-1 and H-2 allow (23) and (25) to be rewritten, respectively as:

$$
\begin{gathered}
\dot{U}_{0}=C_{v} m_{0} \dot{T}_{0} \\
\dot{U}_{0}=C_{p} \dot{m}_{i n}\left(T_{i n}-T_{0}\right)+d Q
\end{gathered}
$$

To simplify, overall in the $1-\mathrm{D}$ model (see next subsection), the temperature dynamics can be expressed in terms of the gas density by introducing the following change of variable (perfect gases law):

$$
\rho_{0}=\frac{p_{i n}}{R T_{0}}
$$

Taking the time derivative of (28) yields

$$
\dot{T}_{0}=-\frac{R}{p_{\text {in }}} T_{0}^{2} \dot{\rho}_{0}
$$

Equalizing (26) and (27) and using (28) - (29) along with the perfect gases law to replace the mass inside the control volume $m_{0}$ in terms of the pressure and the specific gas constant $R$, the following is obtained:

$$
\dot{\rho_{0}}=-\frac{R \gamma T_{i n} \dot{m}_{i n}}{p_{i n} V_{0}} \rho_{0}-\frac{R}{p_{i n} V_{0} C_{v}} \rho_{0} d Q+\frac{\gamma \dot{m}_{i n}}{V_{0}}
$$

where $\rho_{0}=m_{0} / V_{0}$ is the density inside the heating column, $V_{0}$ is the column volume and $\gamma=\frac{C_{p}}{C_{v}}$.

\subsection{Tube Model}

To model the dynamics of the fluid inside the tube, the one-dimensional Euler equations are considered for a perfect gas and a constant tube cross section. These equations can be written in terms of the primitive variables (density $\rho$, particle speed $u$ and pressure $p$ ) as follows (see Winterbone and Pearson [2000]):

$$
\begin{gathered}
\frac{\partial \mathbf{V}}{\partial t}+\mathbf{A}(\mathbf{V}) \frac{\partial \mathbf{V}}{\partial x}+\mathbf{C}(\mathbf{V})=0 \\
\mathbf{V}=\left[\begin{array}{c}
\rho \\
u \\
p
\end{array}\right] ; \mathbf{A}=\left[\begin{array}{ccc}
u & \rho & 0 \\
0 & u & \frac{1}{\rho} \\
0 & a^{2} \rho & u
\end{array}\right] ; \mathbf{C}=\left[\begin{array}{c}
0 \\
G \\
(\gamma-1) \rho(q+u G)
\end{array}\right]
\end{gathered}
$$

where $a=\sqrt{\frac{\gamma p}{\rho}}$ is the speed of sound, $G$ is a term associated with the friction losses and $q$ is a term associated with the wall heat exchanges. In order to simplify (31), the following hypothesis are considered: 
H-21 the propagation speed of the entropy wave (average energy and mass) inside the tube is much slower than the sound speed $u<<a$;

$\mathrm{H}-22$ the pressure inside the tube is considered constant (equal to the atmospheric pressure) because the pressure differential introduced by the fans is very small;

H-23 the heat exchanges and the friction in the tube are neglected: $q=0$ and $G=0$.

Hypothesis H-21 et H-22, imply $\frac{\partial u(x, t)}{\partial x} \approx 0$ and $\frac{\partial p(x, t)}{\partial x}=$ 0 . This reduces system (31) to the following convection equation:

$$
\frac{\partial \rho(x, t)}{\partial t}+u(t) \frac{\partial \rho(x, t)}{\partial x}=0
$$

where $u(t)$ is the time-varying convection parameter of (32). The gas speed $u(t)$ in the tube is measured. Using $\mathrm{H}-21, \mathrm{H}-22$ and $\mathrm{H}-23$ allows the input mass flow rate to be expressed as:

$$
\dot{m}_{i n}=u(t) \rho(0, t) A_{t}
$$

where $A_{t}$ is the tube cross section area. With (33), the boundary conditions of (32) can be expressed as:

$$
\begin{aligned}
& \dot{\rho_{0}}=-\frac{R \gamma T_{i n} u(t) \rho(0, t) A_{t}}{p_{i n} V_{0}} \rho_{0}-\frac{R}{p_{i n} V_{0} C_{v}} \rho_{0} d Q \\
& +\frac{\gamma u(t) \rho(0, t) A_{t}}{V_{0}} ; \quad \rho(0, t)=\rho_{0}
\end{aligned}
$$

\section{OUTPUT TEMPERATURE BOUNDARY CONTROL}

The output temperature boundary control is designed for (32) with boundary conditions (34). Let define the density error as:

$$
\xi=\rho-\rho_{\text {ref }}
$$

where $\rho_{\text {ref }}$ is the desired output density. It is easy to show that system (32) with boundary conditions (34) can be expressed in an LPV form as follows:

$$
\frac{\partial \xi(x, t)}{\partial t}+\Lambda(\varphi) \frac{\partial \xi(x, t)}{\partial x}=0
$$

with boundary conditions:

$$
\dot{\xi_{0}}=A_{c}(\varphi) \xi_{0}+B_{c}(\varphi) d Q+E_{c}(\varphi)
$$

and with the varying parameters defined as follows:

$$
\varphi_{1}=u(t), \quad \varphi_{2}=\rho_{0}, \quad \varphi_{3}=\rho_{0} u(t)
$$

Only $\varphi_{1}$ is measured. $\varphi_{2}$ and $\varphi_{3}$ are considered as uncertain parameters as no temperature measurement is taken inside the heating column. Strictly speaking, system (37) is quasi-LPV because one of the parameters is a state. However, as $\rho_{0}$ can be easily bounded from the knowledge of the operating conditions of the experimental setup, then the system can be considered as an LPV one. Let define the control input as:

$$
d Q=F\left(\varphi, \rho_{r e f}\right)+K \xi(L, t)
$$

where $F\left(\varphi, \rho_{\text {ref }}\right)$ is defined as:

$F\left(\varphi, \rho_{r e f}\right)=-B_{c}(\varphi)^{-1} E_{c}(\varphi)=C_{v} \gamma u(t) A_{t}\left(\frac{p_{i n}}{R}-T_{i n} \rho_{r e f}\right)$

This yields to the system (32) with boundary conditions (34), which corresponds to the system considered in Theorem 1. Note that $d Q$ is independent of the uncertain parameters $\varphi_{2}$ and $\varphi_{3}$, which is crucial for the boundary control implementation. Let define the convex subset $Z_{\varphi}$ in order to operate the experimental setup between the temperatures of $290 \mathrm{~K}$ and $323 \mathrm{~K}$ and a flow speed between $0.63 \mathrm{~m} / \mathrm{s}$ and $3.82 \mathrm{~m} / \mathrm{s}$ :

$$
\begin{aligned}
Z_{\varphi}:= & \left\{\left[\varphi_{1}, \varphi_{2}, \varphi_{3}\right]^{T} \in \mathbb{R}^{3}, \varphi_{1} \in[0.63,3.82],\right. \\
& \left.\varphi_{2} \in[0.968,1.08], \varphi_{3} \in[0.61,4.12]\right\}
\end{aligned}
$$

In order to design the boundary control for system (32) with boundary conditions (34), consider the control architecture presented in Figure 3.

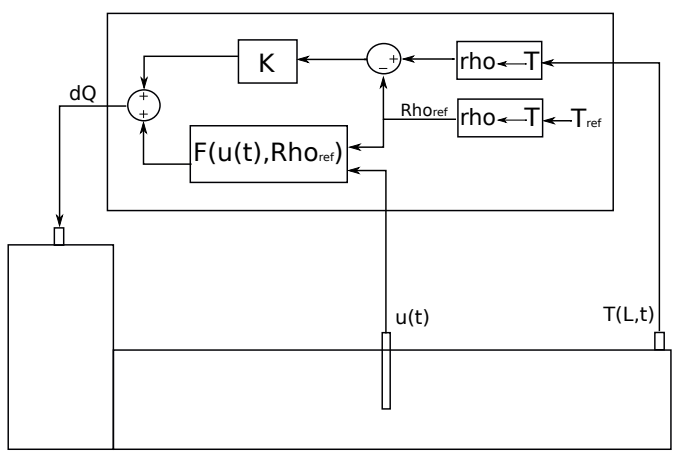

Fig. 3. Flow Tube Control Architecture

Consider the following system parameters: $p_{\text {in }}=1 \times 10^{5}$ $\mathrm{Pa}, \mathrm{T}_{\text {in }}=300 \mathrm{~K}, V_{0}=4 \times 10^{-3} \mathrm{~m}^{3}, A_{t}=6.4 \times$ $10^{-3} \mathrm{~m}^{2}$, and $L=1.5 \mathrm{~m}$ where $L$ is the tube's length. Applying Theorem 1 leads to the following control gain and Lyapunov function parameter $P$ :

$$
K=-654, \quad P=1
$$

which ensure that the equilibrium $\xi=0$ is exponentially stable $\forall \varphi \in Z_{\varphi}$. In order to illustrate the effectiveness of the proposed boundary control strategy, some simulation results of system (32) with boundary conditions (34) and the boundary control (42) are presented for different flow speeds. The results obtained are presented in Figures 4 and 5 . A change of temperature reference from $300 K$ to $320 K$ ( which can be transformed into a density reference using the perfect gases law) is introduced at $1 \mathrm{~s}$.

As depicted in Figure 4, the system effectively follows the change of reference for the different flow speeds. The faster the flow speed, the faster the convergence, 


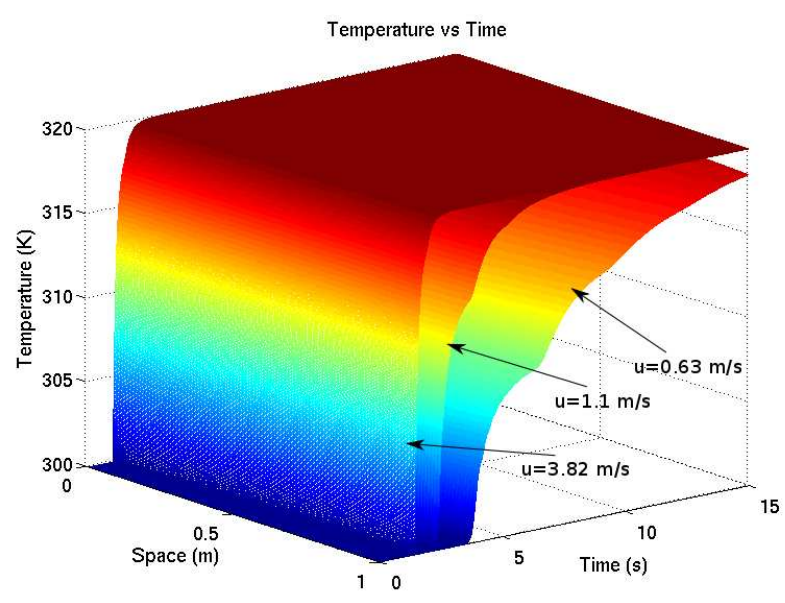

Fig. 4. Output temperature boundary control results for 3 different flow speed

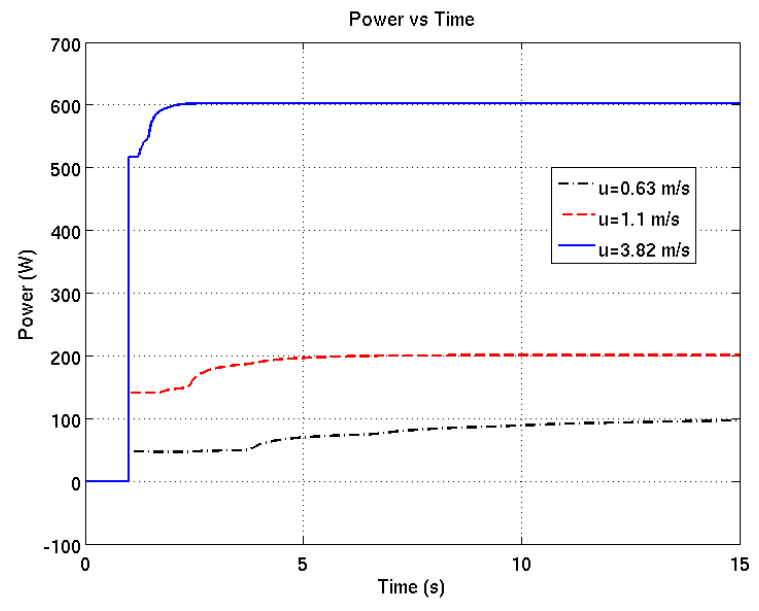

Fig. 5. Control Input for 3 different flow speed

as the fluid transport time is smaller. Figure 5 shows the respective control inputs obtained for the simulation results. It appears that the power dissipated by the heating resistor has to be greater as the flow speed increases. This is due to the fact that in this case, the gas residence time inside the heating column is smaller and the amount of energy absorbed by the gas is less important.

\section{CONCLUSIONS}

This paper was concerned with the boundary control of first order LPV hyperbolic systems with dynamics associated with the boundary conditions. Some sufficient conditions for exponential stability of such systems have been obtained and presented using an LMI approach. The exponential stability has been demonstrated by means of an extended strict Lyapunov function formulation. The modeling of an experimental setup has been developed using a $0-\mathrm{D}$ modeling approach for the heating column and a 1-D modeling strategy for the tube. It has been shown that the experimental setup model can be expressed in an LPV hyperbolic form with dynamic boundary conditions. Simulation results have shown the effectiveness of the contributions presented in this work.
The present results have many applications in different systems governed by hyperbolic PDE's. However, many questions are still open. In particular, a generalization of some sufficient conditions for exponential stability of hyperbolic systems with non-linear dynamic boundary conditions seems to be a challenging issue. Considering perturbations in system (1) and in the boundary conditions dynamics (4) seems to be a natural extension of this work.

\section{REFERENCES}

G. Angelis. System Analysis, Modelling and Control with Polytopic Linear Models. PhD Thesis. Technische Universiteit Eindhoven, Eindhoven, 2001.

G. Bastin, J-M. Coron, and B. d'Andréa Novel. Using hyperbolic systems of balance laws for modelling, control and stability analysis of physical networks. Lecture notes for the Pre-Congress Workshop on Complex Embedded and Networked Control Systems, 17th IFAC World Congress, Seoul, Korea, 2008.

G. Bastin, J-M. Coron, and B. d'Andréa Novel. On Lyapunov stability of linearized Saint-Venant equations for a sloping channel. Networks and Heterogeneous Media., 4(2):177-187, 2009.

F. Castillo, E. Witrant, and L. Dugard. Contrôle de température dans un flux de Poiseuille. IEEE Conférence Internationale Francophone d'Automatique, Grenoble, France, 2012.

F. Castillo, W. Witrant, C. Prieur, and L. Dugard. Dynamic boundary stabilization of linear and quasi-linear hyperbolic systems. 51st IEEE Control and Decision Conference, 2013.

J-M. Coron, G. Bastin, and B. d'Andréa Novel. A strict Lyapunov function for boundary control of hyperbolic systems of conservation laws. IEEE Transactions on Automatic control, 52:2-11, 2007.

J-M. Coron, G. Bastin, and B. d'Andréa Novel. Dissipative boundary conditions for one-dimensional nonlinear hyperbolic systems. SIAM J. Control Optim, 47:14601498, 2008.

I. Kmit. Classical solvability of nonlinear initial-boundary problems for first-order hyperbolic systems. International Journal of Dynamical Systems and Differential Equations Vol. 1, No.3, pages 191-195, 2008.

C. Prieur, J. Winkin, and G. Bastin. Robust boundary control of systems of conservation laws. Mathematics of Control, Signals, and Systems, 20(2):173-197, 2008.

V. Dos Santos and C. Prieur. Boundary control of open channels with numerical and experimental validations. IEEE Transactions on Control Systems Technology, 16(6):1252-1264, 2008.

DE. Winterbone and RJ. Pearson. Theory of Engine Manifold Design: Wave Action Methods for IC Engines. Society of Automotive Engineers. Inc, 2000.

E. Witrant, A. D'Innocenzo, G. Sandou, F. Santucci, M. D. Di Benedetto, A. J. Isaksson, K. H. Johansson, S.-I. Niculescu, S. Olaru, E. Serra, S. Tennina, and U. Tiberi. Wireless ventilation control for large-scale systems: the mining industrial case. International Journal of Robust and Nonlinear Control, 20:226-251, Jan. 2010 . 